Full Length Article

\title{
Facile and efficient in situ synthesis of silver nanoparticles on diverse filtration membrane surfaces for antimicrobial performance
}

\author{
Longbin $\mathrm{Qi}^{\mathrm{a}}{ }^{\mathrm{b}, 1}$, Zhongyun Liu ${ }^{\mathrm{b}, 1}$, Ning Wang ${ }^{\mathrm{b}}$, Yunxia $\mathrm{Hu}^{\mathrm{a}, *}$ \\ a State Key Laboratory of Separation Membranes and Membrane Processes, National Center for International Research on Membrane Science and Technology, School of \\ Materials Science and Engineering, Tianjin Polytechnic University, Tianjin 300387, PR China \\ ${ }^{\mathbf{b}}$ CAS Key Laboratory of Coastal Environmental Processes and Ecological Remediation, Yantai Institute of Coastal Zone Research, Chinese Academy of Sciences, Yantai, \\ Shandong Province 264003, PR China
}

A R T I C L E I N F O

\section{Keywords:}

Silver nanoparticles

Dopamine

Filtration membrane

Antibacterial property

\begin{abstract}
A B S T R A C T
Membrane biofouling remains a major limiting factor for the membrane-based water purification technology. The development of antibacterial membrane surfaces is very important for membrane biofouling mitigation. In this study, we developed a one-pot method of synthesizing silver nanoparticles (Ag NPs) and immobilizing them onto membranes via simply soaking the membranes in a mixture solution of silver nitrate, poly (ethylene glycol) methyl ether thiol (mPEG-SH) and dopamine. Results illustrate that mPEG-SH works as a ligand to slow down the reduction process of silver ions by dopamine for the synthesis of Ag NPs in a controllable manner and also the prevention of Ag NPs aggregation in solution. Moreover, the Ag NPs could be in situ immobilized onto polysulfone ultrafiltration membrane, glass fiber membrane and stainless steel, regardless of their surface properties, but the size of Ag NPs was affected by the substrates. In addition, the Ag NPs immobilization on the polysulfone ultrafiltration membranes increased the bovine serum albumin rejection rate of membrane by $16 \%$, but decreased membrane permeability by $14 \%$ compared to that of the pristine membranes. The Ag NPs containing membranes exhibited outstanding antibacterial properties with more than $90 \%$ antibacterial efficiency against both Gram-negative bacteria Escherichia coli and Gram-positive bacteria Staphylococcus aureus. During the filtration test, the silver release from the Ag NPs containing polysulfone ultrafiltration membranes was very slow and the total accumulation of silver ions released from the membranes was only $7 \%$ of its initial Ag NPs loading after $24 \mathrm{~h}$ filtration operation. The silver ion concentration in the permeate water was $0.35 \pm 0.16 \mathrm{ppb}$, far below the maximal contaminant limit of silver ions in drinking water with no risk for the application of Ag NPs incorporated membranes to treat water. Our work provides a facile and universal approach of synthetizing and simultaneously immobilizing Ag NPs onto diverse membranes for antibacterial properties.
\end{abstract}

\section{Introduction}

Membrane-based separation technology has become one of the most effective and efficient technologies for water purification and wastewater treatment $[1,2]$. However, membrane biofouling remains a major limiting factor for their widespread application [3-5]. Membrane biofouling involving the attachment of microorganisms on membrane surfaces, their growth and development into biofilm significantly decreases the separation performance of membrane and shortens the membrane life time [6]. Importantly, the biofilm and produced extracellular polymeric substances (EPS) are mainly responsible for the membrane water flux decline since it strongly binds on the membrane surfaces to block membrane pores and increase water transport resistance $[7,8]$. Therefore, antibacterial surface modification of filtration membrane is urgently required to suppress biofilm formation on membrane surface.

The incorporation of biocides on membrane surfaces has been proved as an effective approach for antibacterial surface modification and membrane biofouling mitigation since biocides can inactivate bacteria, decrease EPS production and prevent biofilm formation [9]. The incorporated effective biocides on membrane surfaces include metal-based nanoparticles (e.g., silver nanoparticles (Ag NPs) and titanium dioxide), cationic polymers and other nanomaterials such as graphene oxide (GO) and carbon nanotubes (CNTs) [9-14]. Particularly, Ag NPs has been widely used as an effective broad-spectrum biocide to functionalize filtration membrane surfaces for biofouling

\footnotetext{
* Corresponding author.

E-mail address: yunxiahu@yic.ac.cn (Y. Hu).

${ }^{1}$ Longbin Qi and Zhongyun Liu have contributed equally to this work.
} 
mitigation [15-17]. The antibacterial mechanism of Ag NPs, as reported in literatures, is the release of silver ions that can induce cell membrane damage, promote generation of reactive oxygen species (ROS) and disrupt ATP production and DNA replication, ultimately causing the death of bacteria [18-20]. The release and diffusion of silver ions from Ag NPs is a key to prevent the development and growth of biofilm on membrane surfaces since the bacteria in the water solution is inevitably deposited on the membrane surface under the filtration conditions but silver ions can diffuse across the top surface of biofilm to kill the deposited bacteria [21]. In contrast, the biocides with no diffusion capability have a limited membrane biofouling mitigation performance since they cannot effectively kill the deposited and accumulated bacteria on the top surface of biofilm and thus could not efficiently prevent the biofilm growth [22].

Up to date, a variety of methods have been developed to immobilize Ag NPs onto membrane surfaces including blending, depositing, coating and chemical binding [14,23-30]. Liu et al. had developed a method for loading Ag NPs on the alkaline treated polyacrylonitrile membranes via in situ reduction of silver ions to form Ag NPs on membrane surface [27]. This approach is simple, but the incorporated Ag NPs may easily leach from the membranes over time under the cross-flow condition due to the weak interaction between membrane and Ag NPs. The incorporated Ag NPs via chemical binding exhibit excellent stability. For example, Yin et al. had developed anti-fouling thin-film composite membranes by grafting Ag NPs onto the polyamide surface using cysteamine as a bridging agent [31]. However, this approach of Ag NPs immobilization is highly dependent on the membrane surface properties, requiring complicated and time-consuming surface treatments. Recently, a mussel-inspired strategy has been developed as a universal and applicable approach for immobilizing Ag NPs onto various substrates. Polydopamine (PDA) from the oxidative self-polymerization of dopamine (DOPA) can tightly adhere to most of the substrate surfaces [32]. Furthermore, PDA coatings can serve as a versatile platform for secondary surface functionalization thanks to its catechol and amine groups [33]. The catechol groups of PDA have been demonstrated to reduce silver ions to form well-dispersed Ag NPs on the substrate surfaces. Several research groups have successfully synthesized Ag NPs in situ on membrane surfaces via the PDA deposition and the Ag NPs generation, achieving antibiofouling performances [16,34,35]. However, this method of immobilizing Ag NPs through the PDA coating requires two steps including the slow polymerization of dopamine on the substrate surface, and the subsequential generation of Ag NPs upon incubation with silver salt solution, which is a long time process limiting its practical applications on a large scale. Therefore, it remains a tremendous challenge to develop a facile and universal strategy for $\mathrm{Ag}$ NPs immobilization on various filtration membranes.

Herein, for the first time, we report a one-step facile and universal method of synthesizing Ag NPs and in situ immobilizing them onto filtration membranes upon simply soaking in a mixture solution of silver nitrate, poly (ethylene glycol) methyl ether thiol (mPEG-SH) and DOPA. Polysulfone ultrafiltration membrane, glass fiber membrane and stainless steel were selected as representative organic, inorganic, and metal substrates, respectively, and Ag NPs were in situ immobilized on their surfaces. The impact of Ag NPs immobilization on membrane filtration properties was investigated using the polysulfone ultrafiltration membrane as a model, and the silver ions leaching from the polysulfone membrane surface was measured during the filtration measurements. Importantly, the antibacterial properties of Ag NPs containing membranes were investigated using the diffusion inhibition zone (DIZ) method, the colony forming unit (CFU) method, and the live/dead assay. Gram-negative E. coli and Gram-positive $S$. aureus were selected as model strains.

\section{Materials and methods}

\subsection{Materials}

Dopamine (DOPA), polysulfone (PSF, $\mathrm{M}_{\mathrm{n}}$ : 22,000 Da), poly (ethylene glycol) methyl ether thiol (mPEG-SH, $\mathrm{M}_{\mathrm{n}}$ : 1000), bovine serum albumin (BSA, $\mathrm{M}_{\mathrm{n}}: 67,000 \mathrm{Da}$ ) and propidium iodide (PI) stain were purchased from Sigma Aldrich (St. Louis, MO, USA). SYTO ${ }^{\circledR} 9$ green fluorescent nucleic acid stain was obtained from Invitrogen (Eugene, Oregon, USA). Silver nitrate $\left(\mathrm{AgNO}_{3}\right.$, analytical purity) was received from Sinopharm Chemical Reagent Beijing Co., Ltd, China. Escherichia coli (E. coli, DH5 $\alpha$ ) was purchased from Beijing Dingguo Changsheng Biotechnology Co., Ltd., China. Staphylococcus aureus (S. aureus, CICC 10201) was obtained from China Center of Industrial Culture Collection.

Polysulfone ultrafiltration (UF) membrane was fabricated by nonsolvent induced phase separation method following the reported protocol [36]. The glass fiber membrane with $0.7 \mu \mathrm{m}$ average pore size and $420 \mu \mathrm{m}$ thickness was purchased from GE Healthcare (GE, NJ, USA). The commercial stainless steel (ZR-030, 400 mesh, $30 \mu \mathrm{m}$ wire diameter) was supplied from Zhongrui wire mesh industry Co., Ltd, China.

\subsection{One-step synthesis and immobilization of $\mathrm{Ag} N \mathrm{~N}$ s on filtration membranes}

Ag NPs were synthesized using DOPA as a reducing agent and mPEG-SH as a stabilizing agent. A typical procedure is as follows: $10 \mathrm{mg}$ of mPEG-SH was dissolved in $100 \mathrm{~mL} 20 \mathrm{mM} \mathrm{AgNO}_{3}$ aqueous solution and incubated for $10 \mathrm{~min}$ open to air at room temperature under $60 \mathrm{rpm}$ shaking in a shaker (Jintan Science Analysis Instrument Co., Ltd., China). Then, $2 \mathrm{~mL}$ of $0.1 \mathrm{~g} \mathrm{~mL}^{-1}$ DOPA aqueous solution was added dropwise into the $\mathrm{AgNO}_{3}$ solution to reduce silver ions and the mixture solution was incubated for two hours. The formation and growth of $\mathrm{Ag}$ NPs was monitored using a UV-visible spectrophotometer (UV-Vis, TU1810 , Persee, China) with a scan range of $200-800 \mathrm{~nm}$. Ag NPs were collected through $20 \mathrm{~min}$ centrifugation at $140,000 \mathrm{rpm}$, washed three times with deionized water, vacuum dried and finally stored at $4{ }^{\circ} \mathrm{C}$. In order to determine the roles of $\mathrm{AgNO}_{3}$, DOPA and mPEG-SH in the formation process of $\mathrm{Ag} \mathrm{NPs}$, three mixture solutions without $\mathrm{AgNO}_{3}$, or DOPA, or mPEG-SH, were used as negative controls respectively, shown in Table S1.

Synthesis and immobilization of Ag NPs on membranes was performed via a simple dip-coating method. PSF UF membrane, glass fiber membrane and stainless steel were used as model substrates. Briefly, $10 \mathrm{mg}$ of mPEG-SH was dissolved in $100 \mathrm{~mL} 20 \mathrm{mM} \mathrm{AgNO}_{3}$ aqueous solution and then the membrane coupons were immersed in the solution and incubated for $10 \mathrm{~min}$ open to air at room temperature under $60 \mathrm{rpm}$ shaking. After that, $2 \mathrm{~mL}$ of $0.1 \mathrm{~g} \mathrm{~mL}^{-1}$ DOPA aqueous solution was added dropwise into the mixture solution. $2 \mathrm{~h}$ later, the membrane coupons were rinsed with deionized water three times, dried at ambient conditions and stored in a light-proof dark chamber for further characterization.

\subsection{Characterization}

The morphologies of Ag NPs and Ag NPs modified membranes were observed using a scanning electron microscope (SEM, S-4800, Hitachi, Japan) and a transmission electron microscope (TEM, H7650, Hitachi, Japan). The elemental compositions of Ag NPs were characterized using X-ray photoelectron spectroscopy (XPS, Thermo Escalab 250Xi, Thermo Fisher Scientific, USA) with monochromatic Al-Ka X-ray source ( $h v=1486.6 \mathrm{eV}$ ). All binding energies were referenced to that of the neutral C $1 \mathrm{~s}$ hydrocarbon peak at $284.6 \mathrm{eV}$. Survey spectra over $0-1200 \mathrm{eV}$ were obtained with a step size of $1 \mathrm{eV}$. The average hydrodynamic diameter distribution of nanoparticles was measured using a Zetasizer Nano ZS dynamic light scattering (DLS) system (ZS90, 
Malvern, UK). The FT-IR analysis was performed with a Fourier Transform Infrared Spectrometer (FT/IR-4100, Jasco, Japan). Thermogravimetric analysis (TGA) was performed using a thermal analyzer (Mettler 5MP/PF7548/MET/400W, Mettler Toledo Co., Switzerland). Samples were heated to $200^{\circ} \mathrm{C}$ from room temperature and incubated for $10 \mathrm{~min}$ to remove moisture, and then heated up to $800{ }^{\circ} \mathrm{C}$ in air with a heating increase rate of $20^{\circ} \mathrm{C} \mathrm{min}^{-1}$. The membrane surface charges were measured using Anton Paar SurPASSTM 2 electrokinetic analyzer (Anton Paar, Austria) using $1 \mathrm{mM}$ phosphate buffered saline as a background electrolyte solution.

\subsection{Antibacterial activity tests of Ag NPs generated membranes.}

Antimicrobial activities of Ag NPs generated membranes were assessed using both the colony forming unit (CFU) method and the diffusion inhibition zone (DIZ) method [17,21]. A Gram-negative E. coli and a Gram-positive $S$. aureus were used as model strains. The CFU experiments were performed following the protocol reported in our previous study [34]. Briefly, circular membrane coupons with $1.6 \mathrm{~cm}$ in diameter were incubated with $20 \mathrm{~mL}$ physiological saline solution $\left(0.15 \mathrm{M} \mathrm{NaCl}, 20 \mathrm{mM} \mathrm{NaHCO}_{3}, \mathrm{pH}\right.$ 7.0) containing bacteria $\left(\sim 10^{7} \mathrm{CFU} \mathrm{mL}{ }^{-1}\right)$ for $5 \mathrm{~h}$ at $37^{\circ} \mathrm{C}$. After incubation, the membrane coupons were gently rinsed with deionized water and then bath-sonicated for $7 \mathrm{~min}$ in $10 \mathrm{~mL}$ of physiological saline solution to remove the attached bacteria. The bacteria suspension was serially diluted, plated on LB agar plates and incubated overnight at $37^{\circ} \mathrm{C}$ following which bacteria colonies were counted and their antibacterial efficiencies were calculated. Three replicates were performed and the results were reported as the average value with standard deviation. For DIZ tests, 100 $\mu \mathrm{L}$ of bacterial culture were spread onto an LB agar plate. The circular membrane coupons were then placed onto the plates and incubated overnight at $37^{\circ} \mathrm{C}$. The DIZs formed around the membrane coupons were observed and photographed.

The bacteria adhesion on the surfaces of PSF UF membrane was investigated using a live/dead assay following our previous protocol with minor change [37]. Circular PSF UF membrane coupons were immersed in $20 \mathrm{~mL}$ of $E$. coli suspensions $\left(\sim 10^{7} \mathrm{CFU} \mathrm{mL}^{-1}\right)$ in LB broth and incubated at $37^{\circ} \mathrm{C}$ for $24 \mathrm{~h}$. Then the circular membrane coupons were gently rinsed with sterile physiological saline solution and stained using $3.34 \mu \mathrm{M}$ SYTO ${ }^{\circledR 9}$ and $20 \mu \mathrm{M}$ PI for 15 min in dark. After rinsed twice with deionized water, the membrane coupons were visualized using confocal laser scanning microscope (CLSM, Fluoview-FV1000, Olympus, Japan). Two sets of lasers were used for the excitation of SYTO $^{\circledR} 9$ and PI including the argon laser (excitation at $488 \mathrm{~nm}$ and emission at $503 \mathrm{~nm}$ ) and the diode-pumped solid state laser (excitation at $559 \mathrm{~nm}$ and emission at $619 \mathrm{~nm}$ ).

\subsection{Membrane filtration tests}

The PSF UF membrane was selected as a model to investigate the impact of Ag NPs immobilization on membrane filtration performance. In a typical procedure, the membrane coupon was mounted on a deadend filtration cell (Amicon 8010, Millipore) with an effective area of $3.85 \mathrm{~cm}^{2}$. Pre-compacted with DI water at $1.5 \mathrm{bar}$ for $30 \mathrm{~min}$, the membrane water flux was subsequently measured at 1.0 bar for $30 \mathrm{~min}$. The permeate water weight was continuously monitored using an electronic balance (ME3002, Mettler, Switzerland). The pure water flux was calculated according to the following Eq. (1):

$J=\frac{\mathrm{m}}{\rho \mathrm{St}}$

where $\mathrm{J}$ is the membrane pure water flux $\left(\mathrm{LMH} \mathrm{bar}^{-1}\right.$, $\left.\mathrm{L} \mathrm{m}^{-2} \mathrm{~h}^{-1} \mathrm{bar}^{-1}\right)$; $\mathrm{m}$ is the permeate water weight $(\mathrm{g}) ; \rho$ is DI water density $\left(\mathrm{g} \mathrm{cm}^{-3}\right) ; \mathrm{S}$ is the effective membrane filtration area $\left(\mathrm{m}^{2}\right)$.

The membrane selectivity was tested using $1 \mathrm{~g} \mathrm{~L}^{-1}$ bovine serum albumin (BSA) aqueous solution (PBS, pH 7.4) as the feed at 1.0 bar. The BSA concentration in the permeate solution was measured using a $\mathrm{UV}$-vis spectrophotometer at $280 \mathrm{~nm}$. The BSA rejection was calculated according to the following Eq. (2)

$R=\left(1-\frac{C_{t}}{C_{0}}\right) \times 100 \%$

where $\mathrm{R}$ is the BSA rejection rate; $\mathrm{C}_{t}$ and $\mathrm{C}_{0}$ are the concentration of $\mathrm{BSA}$ in the permeate and the feed solutions, respectively.

\subsection{Quantification of silver loading and silver ions release}

To quantify the silver mass loadings on membranes, the Ag NPs modified membrane coupons $\left(2.0 \mathrm{~cm}^{2}\right)$ were immersed in $10 \mathrm{~mL} 3.5 \%$ $\mathrm{HNO}_{3}$ solution for $48 \mathrm{~h}$ to dissolve the Ag NPs completely following our previous protocol [34]. Subsequently, the dissolved silver ions in the solution were analyzed by an inductively coupled plasma mass spectrometry (ICP-MS, ELAN DRC II, PerkinElmer (Hong Kong) Ltd.). The silver mass loadings on membranes were quantified according to the obtained concentration of silver ions and the surface area of membranes. The average value was reported with the standard deviation as the final result measured from at least three individual specimens.

The silver leaching was evaluated during the filtration process. Ultrapure water was filtered through the Ag NPs modified PSF UF membranes $\left(3.85 \mathrm{~cm}^{2}\right)$ in a dead-end filtration cell at 1.0 bar for $24 \mathrm{~h}$. At the predetermined time intervals, $10 \mathrm{~mL}$ of the filtrate was taken out and acidified with $0.33 \mathrm{~mL}$ of $68 \%$ nitric acid for ICP-MS analysis. The released silver ions amount was calculated according to the obtained concentration of silver ions and the volume of filtrate. After the filtration test, the Ag NPs incorporated PSF UF membranes were analyzed using SEM to investigate the changes of particle size and number. The particle size and number of Ag NPs on the membrane surfaces were measured using Nano Measurer software and Image $\mathrm{J}$ software, respectively.

\section{Results and discussion}

\subsection{Synthesis and characterization of Ag NPs using dopamine as a reducing} reagent

$\mathrm{Ag}$ NPs were successfully synthesized by adding DOPA to $\mathrm{AgNO}_{3}$ solution in the presence of mPEG-SH, as shown in Scheme 1. With the addition of DOPA, the colour of $\mathrm{AgNO}_{3} / \mathrm{mPEG}$-SH mixture turned from

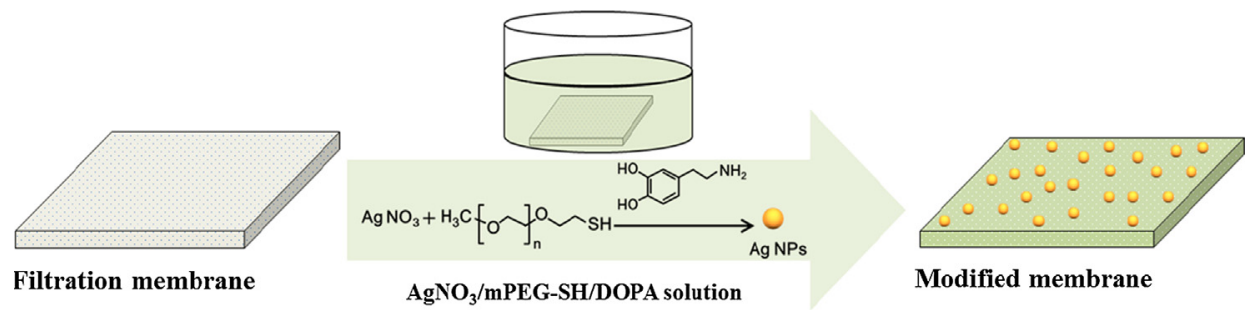

Scheme 1. One-step synthesis and simultaneous immobilization of Ag NPs on the filtration membrane surfaces. 

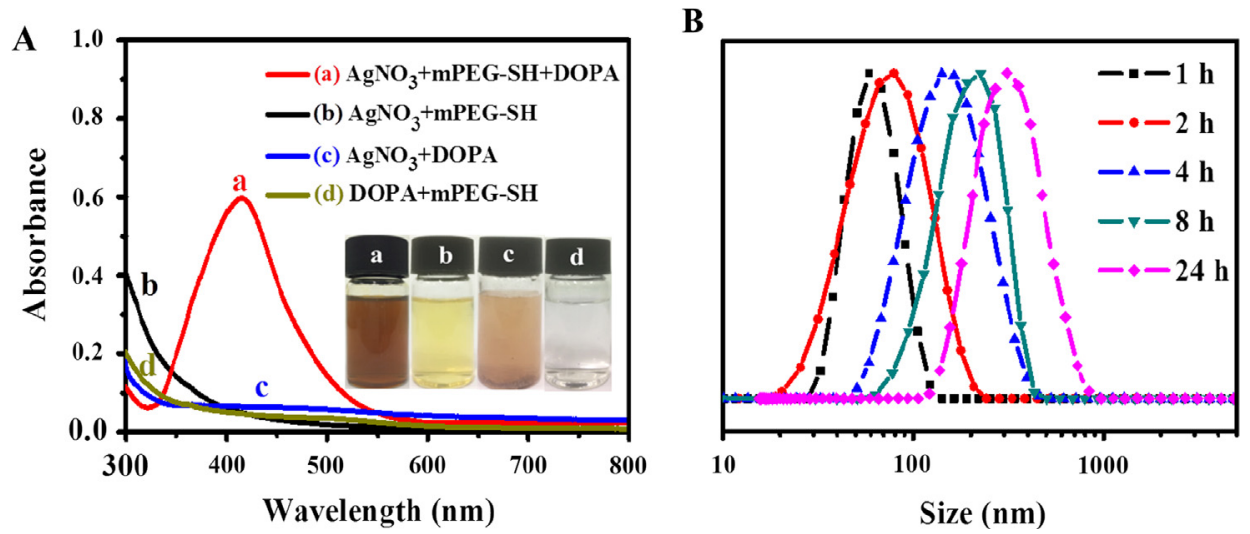

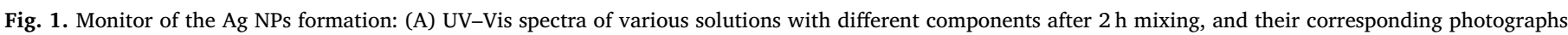
(insert); (B) The hydrodynamic diameter distribution of Ag NPs at different reaction time increasing from $1 \mathrm{~h}, 2 \mathrm{~h}, 4 \mathrm{~h}, 8 \mathrm{~h}$, to $24 \mathrm{~h}$.

light yellow to brown as shown in Fig. 1A, and an intense UV-Vis absorption peak at $412 \mathrm{~nm}$ was observed from the characteristic surface plasmon peak of Ag NPs, indicating the synthesis of Ag NPs. With prolonging the reaction time of $\mathrm{AgNO}_{3} / \mathrm{mPEG}-\mathrm{SH} / \mathrm{DOPA}$ solution from $1 \mathrm{~h}$ to $24 \mathrm{~h}$, the colour of the solution darkened gradually and its UV-Vis absorption peak shifted to longer wavelength from $410 \mathrm{~nm}$ to $433 \mathrm{~nm}$, shown in Fig. S1, indicating the increase of Ag NPs hydrodynamic diameter over time [38]. The hydrodynamic diameter distribution of Ag NPs determined by DLS, shown in Fig. 1B, illustrates that the hydrodynamic diameter of Ag NPs increases quickly from $58.7 \mathrm{~nm}$ to $141.8 \mathrm{~nm}$ during the first $4 \mathrm{~h}$, and then increases slowly to $295.3 \mathrm{~nm}$ with the immersion time increasing to $24 \mathrm{~h}$, further confirming the hydrodynamic diameter increase of Ag NPs over time. A similar trend was reported to confirm the nucleation and growth mechanism of $\mathrm{Ag}$ NPs in the reduction process [39]. During this process, the phenolic hydroxyls of DOPA lost electrons to be oxidized and polymerized, and silver ions were reduced to form Ag NPs [40]. Although DOPA polymerizes generally at alkaline condition, this reaction still occurred in the $\mathrm{AgNO}_{3}$ solution with a $\mathrm{pH}$ of 5.0, which may because of the strong oxidation properties of silver ion, similar as the reported copper ion $[37,41]$.

To determine the crucial role of mPEG-SH in the synthesis of $\mathrm{Ag}$ NPs, a mixture solution of $\mathrm{AgNO}_{3}$ and DOPA was monitored in the absence of mPEG-SH, and bulk grey precipitation was immediately observed at the bottom of the reaction flask as shown in Fig. 1A, because of the rapid aggregation of the formed particles. Furthermore, there was no characteristic absorbance peak of Ag NPs detected from the $\mathrm{AgNO}_{3} / \mathrm{DOPA}$ mixture solution as shown in Fig. 1A, further confirming no Ag NPs formed in solution. Thus, it might demonstrate the predominant role of mPEG-SH as a stabilizer during the synthesis of Ag NPs, since the thiol group of mPEG-SH could chelate silver ions and slow down the reduction process of silver ions by dopamine to synthesize Ag NPs in a controllable manner [42]. Moreover, the Ag NPs solution stored for a week and did not present obvious aggregation using the DLS analysis, as shown in Fig. S3, which is due to the MPEG surrounding Ag NPs preventing their aggregation in solution [43].

The morphologies of Ag NPs were observed using scanning electron microscopy (SEM) and transmission electron microscopy (TEM) to see spherical particles with $90 \mathrm{~nm}$ in average diameter as shown in Figs. 2A and S2, respectively. Elemental analysis was characterized by X-ray photoelectron spectroscopy (XPS) to detect strong silver signals from the nanoparticles, as shown in Fig. 2B. The binding energy peaks at $368 \mathrm{eV}$ for $\mathrm{Ag} 3 \mathrm{~d}_{5 / 2}$ orbital and $374 \mathrm{eV}$ for $\mathrm{Ag} 3 \mathrm{~d}_{3 / 2}$ orbital were clearly observed in high-resolution XPS spectrum of $\mathrm{Ag}$ peaks, further confirming the formation of Ag NPs. To investigate the organic components coated on the Ag NPs surface, the FT-IR spectra of mPEG-SH, PDA and $\mathrm{Ag}$ NPs were collected and compared. The spectrum of the Ag NPs displayed a well-defined peak at $1083 \mathrm{~cm}^{-1}$ arising from the abundant $\mathrm{C}-\mathrm{O}-\mathrm{C}$ stretching vibrations, which was a characteristic peak of mPEG-SH, indicating mPEG-SH is on the surface of Ag NPs. Meanwhile, a weak peak at $1015 \mathrm{~cm}^{-1}$ was observed on $\mathrm{Ag} \mathrm{NPs}$ arising from the $\mathrm{C}=$ $\mathrm{N} \& \mathrm{C}=\mathrm{C}$ stretching vibrations of PDA (Fig. 2C), indicating the PDA exists on the surface of Ag NPs. Thermogravimetric analysis (TGA) was performed to measure the organic and inorganic content of the nanoparticles. The weight loss of $\mathrm{Ag} \mathrm{NPs}$ from $200^{\circ} \mathrm{C}$ to $800^{\circ} \mathrm{C}$ was only $4.1 \%$ of its mass illustrating a low organic content and high silver content in Ag NPs (Fig. 2D). Thus, it can conclude that Ag NPs is covered with small amount of mPEG-SH and PDA.

\subsection{Surface-independent immobilization of Ag NPs on filtration membranes}

Polysulfone (PSF) ultrafiltration (UF) membrane, glass fiber membrane and stainless steel were selected as representative organic, inorganic, and metal substrates, respectively. In situ synthesis and immobilization of Ag NPs on different membranes was performed by immersing the substrates into the mixture solution of $\mathrm{AgNO}_{3} / \mathrm{mPEG}$ $\mathrm{SH} / \mathrm{DOPA}$ for $2 \mathrm{~h}$, the colour of PSF UF membrane and glass fiber membrane turned from pale to brown, and the colour of stainless steel did not change obviously due to its original dark colour, as shown in Fig. S4. Surface morphologies of the membranes were observed using SEM to find numerous small particles generated on all membrane surfaces shown in Fig. 3, similar as the reported morphologies of Ag NPs generated on the membranes through PDA chemistry [34]. The low magnification SEM image of Ag NPs loaded PSF UF membrane illustrates that the Ag NPs uniformly distributed on membrane surface on a relatively large surface area, as shown in Fig. S5. The average diameter of Ag NPs generated on the various membranes was different varying from $35.2 \pm 6.8 \mathrm{~nm}$ on PSF UF membrane to $54.6 \pm 7.2 \mathrm{~nm}$ on stainless steel even under the same reaction conditions (Fig. S6), presenting that the size of Ag NPs is also affected by the substrates, and fibrous surfaces allow for efficient surface exposure of silver nuclei initiated from the membrane to silver salt solution and thus fast growth of Ag NPs. Moreover, the Ag NPs generated on membrane surfaces are smaller than these generated in the bulk solution, which indicates the formation of Ag NPs occurs simultaneously in the solution and on the membrane surface, and also the substrates limit the surface exposure of silver nuclei initiated from the membrane to silver salt solution and thus slow down the growth of Ag NPs on the membrane surface. The surface elements of modified membranes were analyzed using XPS to detect strong silver signals on all modified membranes, as shown in Fig. 3B. The high-resolution XPS spectrum of Ag peaks, shown in Fig. 3C, can be fitted into two different species of $\mathrm{Ag} 3 \mathrm{~d} 5 / 2$ where a strong signal at $368.0 \mathrm{eV}$ is assigned to metallic silver $\mathrm{Ag}(0)$ and a weak signal at $368.7 \mathrm{eV}$ is attributed to $\mathrm{Ag}(+)$ binding with the thiols 
A

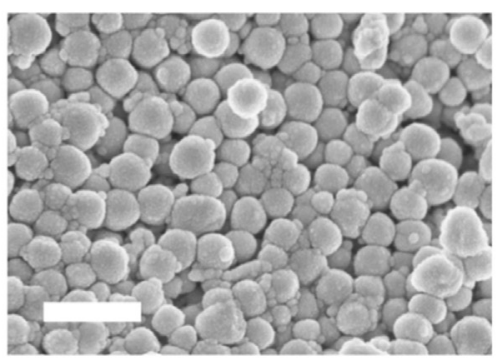

C

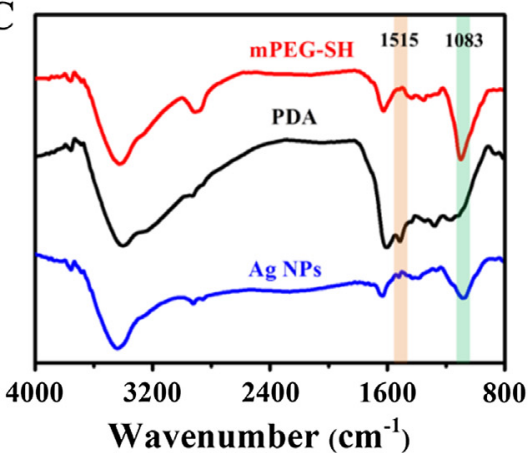

B

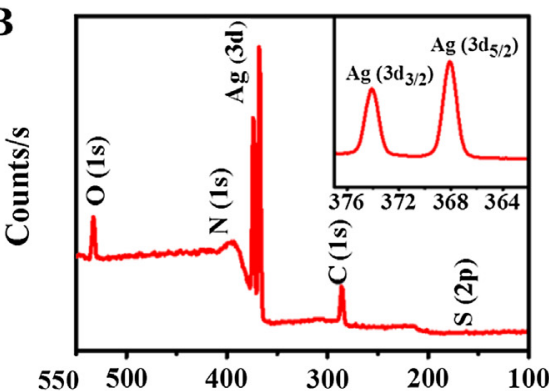

D

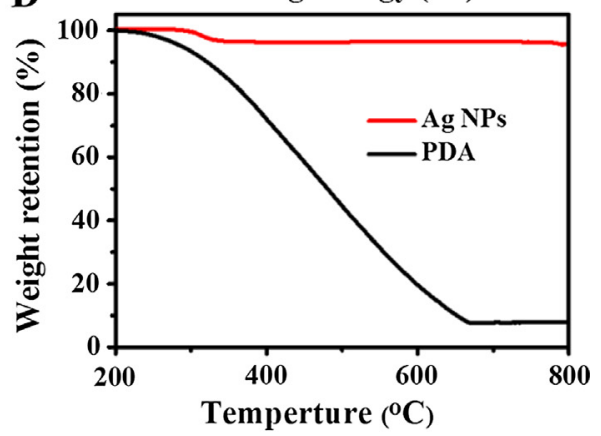

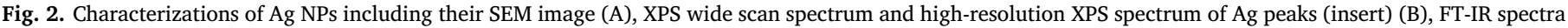
(C) and TGA plots (D). Scale bar in the SEM image is $200 \mathrm{~nm}$.

moieties and PDA $[44,45]$. Meanwhile, the binding energy peaks at $400 \mathrm{eV}$ for N1s were observed indicating the existence of PDA on the membranes. No obvious peak for $\mathrm{S}(2 \mathrm{p})$ was observed for the modified glass fiber membrane and stainless steel except strong $S(2 p)$ peak detected from polysulfone, which might due to very limited amount of
mPEG-SH covered on the modified membranes, and also a tiny amount of sulfur from mPEG-SH even covered on the modified membranes. The surface charges of Ag NPs modified membranes displayed a slight increase but remained negatively charged compared with those of the pristine membranes, as presented in Table S2, indicating that the Ag
A

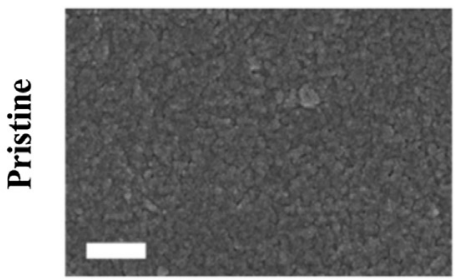

$Z_{0}^{n}$

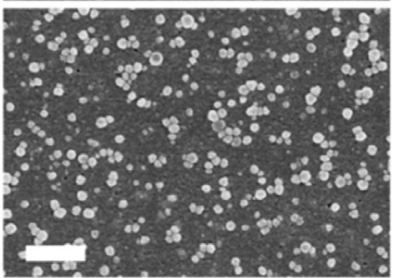

B

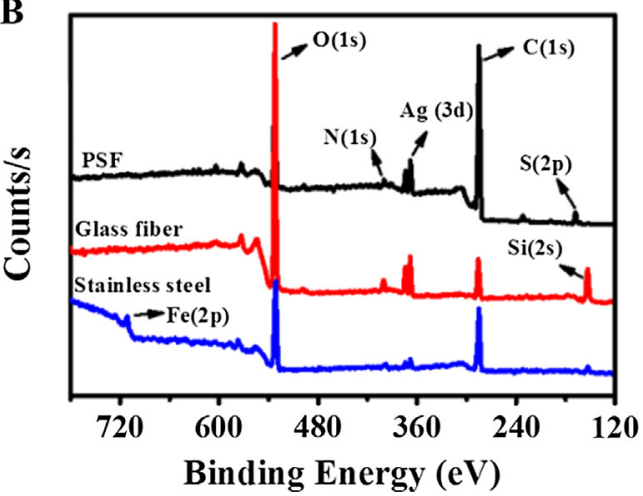

Glass fiber
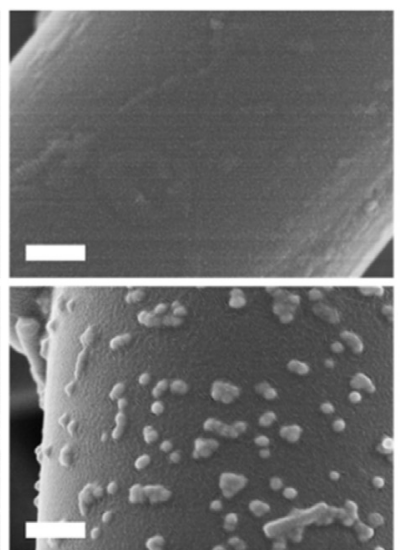

Stainless steel
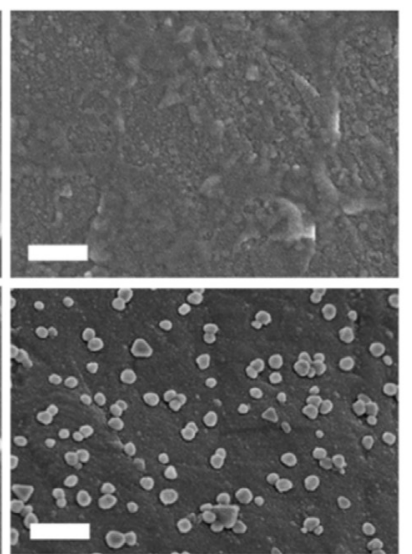

C

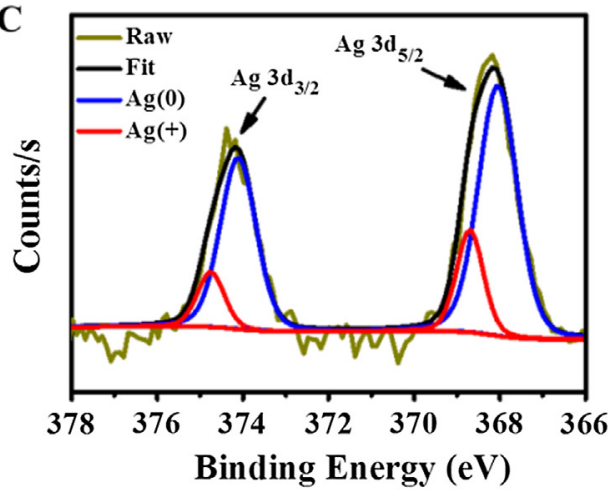

Fig. 3. Characterizations of Ag NPs immobilized membranes: (A) SEM images of PSF UF membrane, glass fiber membrane and stainless steel before and after the synthesis and immobilization of Ag NPs, (B) their corresponding XPS spectra and (C) high-resolution XPS spectrum of Ag peaks. The membranes were immersed in the mixture solution containing $20 \mathrm{mM}$ of $\mathrm{AgNO}_{3}, 1 \mathrm{mM}$ of mPEG-SH and $2 \mathrm{mg} \mathrm{mL}^{-1}$ DOPA for $2 \mathrm{~h}$. Scale bars in all SEM images are $200 \mathrm{~nm}$. 
A

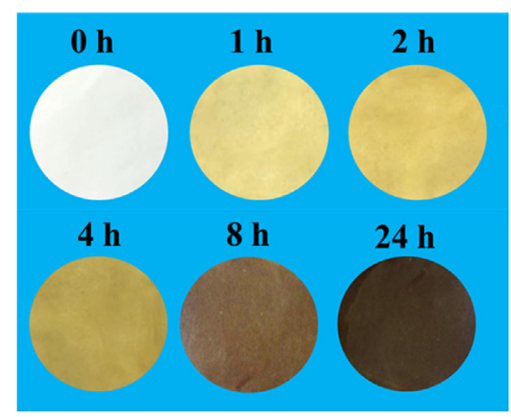

B

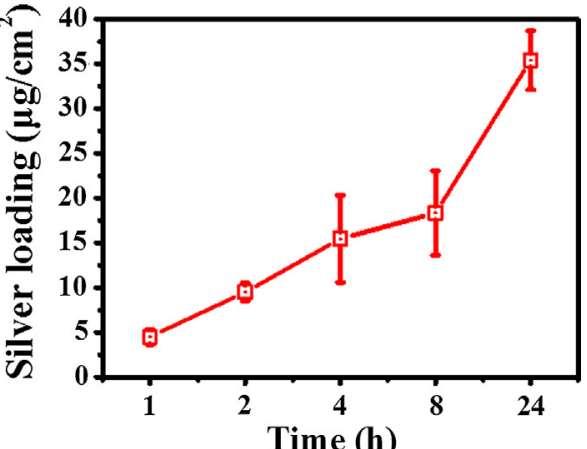

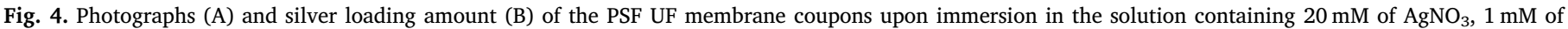
mPEG-SH and $2 \mathrm{mg} \mathrm{mL}^{-1}$ DOPA for $1 \mathrm{~h}, 2 \mathrm{~h}, 4 \mathrm{~h}, 8 \mathrm{~h}$, and $24 \mathrm{~h}$, respectively.

NPs immobilization and thin PDA coating increase the surface charges of polysulfone and stainless steel membranes to a limited range.

It can conclude that Ag NPs could be synthesized in situ and immobilized on diverse membranes through facile one-pot incubation with the mixture solution containing $\mathrm{AgNO}_{3}$, mPEG-SH and DOPA, similar as the PDA coating which can attach on most of substrates owing to its universal adhesive properties [32]. However, the molar fractions of nitrogen elements on the modified substrates $(1.9 \%$ on PSF UF membrane) were lower than that formed through the PDA coating, indicating the very thin coatings of PDA formed on Ag NPs [16,37]. The water contact angle of PSF UF membranes, shown in Fig. S7, did not change much after the modification, confirming the low PDA coating on the substrate which might be beneficial for the alleviation of membrane pore blockage and water flux decline.

Time-dependent loading amount of Ag NPs on the PSF UF membrane was investigated as a representative example. The colour of PSF UF membrane turned from pale to dark brown with prolonging the immersion time in the $\mathrm{AgNO}_{3} / \mathrm{mPEG}$-SH/DOPA solution from $1 \mathrm{~h}$ to $24 \mathrm{~h}$, as shown in Fig. 4A. The silver loading amount was quantitatively characterized using an inductive coupled plasma mass spectrometer (ICP-MS). Results shown in Fig. 4B illustrate that the silver loading on the PSF UF membranes increases quickly from $2.9 \pm 0.2 \mu \mathrm{g} / \mathrm{cm}^{2}$ to $15.4 \pm 4.8 \mu \mathrm{g} / \mathrm{cm}^{2}$ during the first $4 \mathrm{~h}$, and then increases slowly to $35.4 \pm 3.3 \mu \mathrm{g} / \mathrm{cm}^{2}$ with the immersion time increasing to $24 \mathrm{~h}$ correspondingly. Therefore, the silver loading on the substrate could be easily tailored through controlling the immersion time of the substrates in the $\mathrm{AgNO}_{3} / \mathrm{mPEG}-\mathrm{SH} / \mathrm{DOPA}$ solution. Moreover, the silver loading amount on the PSF UF membrane after $24 \mathrm{~h}$ immersion was higher than most of the reported results $[16,17,35]$, suggesting that this synthesis and immobilization of Ag NPs is simple and effective on the various substrates.

\subsection{Ag NPs and Ag NPs containing membranes exhibit strong antibacterial} properties

$\mathrm{Ag}$ NPs have been reported to work as an effective broad-spectrum biocide with low toxicity towards mammalian cells [46,47]. To evaluate the antibacterial activities of the synthesized Ag NPs, E. coil was incubated with various concentrations of Ag NPs and their growth curves were collected. Results shown in Fig. 5A illustrate that the bacterial growth curves strongly depended on the $\mathrm{Ag}$ concentration in the LB medium. Without exposure to Ag NPs, the E. coil reached to exponential phase rapidly in $6 \mathrm{~h}$. When the Ag NPs concentration increased to $30 \mu \mathrm{g} / \mathrm{mL}$, bacterial growth was significantly inhibited, presenting a strong antibacterial performance of Ag NPs. The concentrations of Ag NPs inhibiting the bacterial growth in the experiments is relatively high due to the big size of the synthesized Ag NPs since big Ag NPs may result in a low antibacterial efficiency because of the unefficient exposure of bacteria to Ag NPs and slow release behavior of silver ions $[38,48]$.

The antibacterial properties of Ag NPs containing membranes were investigated using the diffusion inhibition zone (DIZ) method and the colony forming unit (CFU) method, and Gram-negative E. coli and Gram-positive $S$. aureus were selected as model strains. The DIZ images of different membranes were presented in Figs. S8 and S9 to show no antibacterial zone around the pristine membranes, but the clear bacterial-free areas around the Ag NPs containing substrates were observed, confirming the release and diffusion of Ag NPs to kill bacteria. The antibacterial efficiency was further quantified via the CFU method. Compared with their pristine ones, the number of viable $E$. coli cells attached on the Ag NPs containing membranes was decreased by $93.9 \% \pm 1.6 \%, 95.1 \% \pm 1.4 \%$ and $96.8 \% \pm 0.9 \%$ on the PSF UF membranes, glass fiber membranes and stainless steel, respectively, as shown in Fig. 5B. For $S$. aureus, the Ag NPs containing PSF UF membranes, glass fiber membranes and stainless steel also exhibited high antibacterial efficiency as $91.6 \% \pm 3.8 \%, 94.0 \% \pm 5.2 \%$ and $95.4 \% \pm 2.2 \%$ respectively, compared with their pristine samples, as shown in Fig. S10. The live/dead assay was performed to observe live and dead cells on the containing PSF UF membrane surfaces. As shown in Fig. 5C, the green fluorescence from the live bacteria dominated on the pristine PSF UF membrane surface and the red fluorescence from the dead bacteria dominated on the Ag NPs containing PSF UF membrane surface, suggesting that most of the attached bacteria on membrane surfaces were killed and their growth was also prohibited. The dead bacteria can be easily washed off through the cleaning process, which is a key for membrane biofouling mitigation and provide a great benefit for membrane water flux recovery after the cleaning [49]. All of these results demonstrate the immobilization of Ag NPs on membranes impart them outstanding antibacterial properties.

\subsection{The impact of Ag NPs immobilization on membrane filtration properties and silver leaching from membrane surface during the filtration test}

The PSF UF membrane was used as a model to investigate the impact of Ag NPs immobilization on membrane filtration properties. Fig. 6 shows that the water flux of Ag NPs immobilized PSF UF membranes was decreased by $14 \%$ compared to that of the pristine membrane. But, the BSA rejection of the membrane was increased from $42 \%$ for the pristine membrane to $58 \%$ for Ag NPs immobilized membranes. These results illustrate that the Ag NPs immobilization on the PSF UF membranes increased the BSA rejection from the decreased surface pore size, but decreased membrane water flux due to the increased water transport resistance.

The silver ions leaching from the Ag NPs containing PSF UF membranes were evaluated in a dead-end filtration system and the silver ions in the permeate were quantified by ICP-MS. The silver ion concentration in the permeate was $2.71 \pm 0.42 \mathrm{ppb}$ in the first $1 \mathrm{~h}$ of test, dramatically decreasing to below $1 \mathrm{ppb}$ within the $4 \mathrm{~h}$ of test, and then 

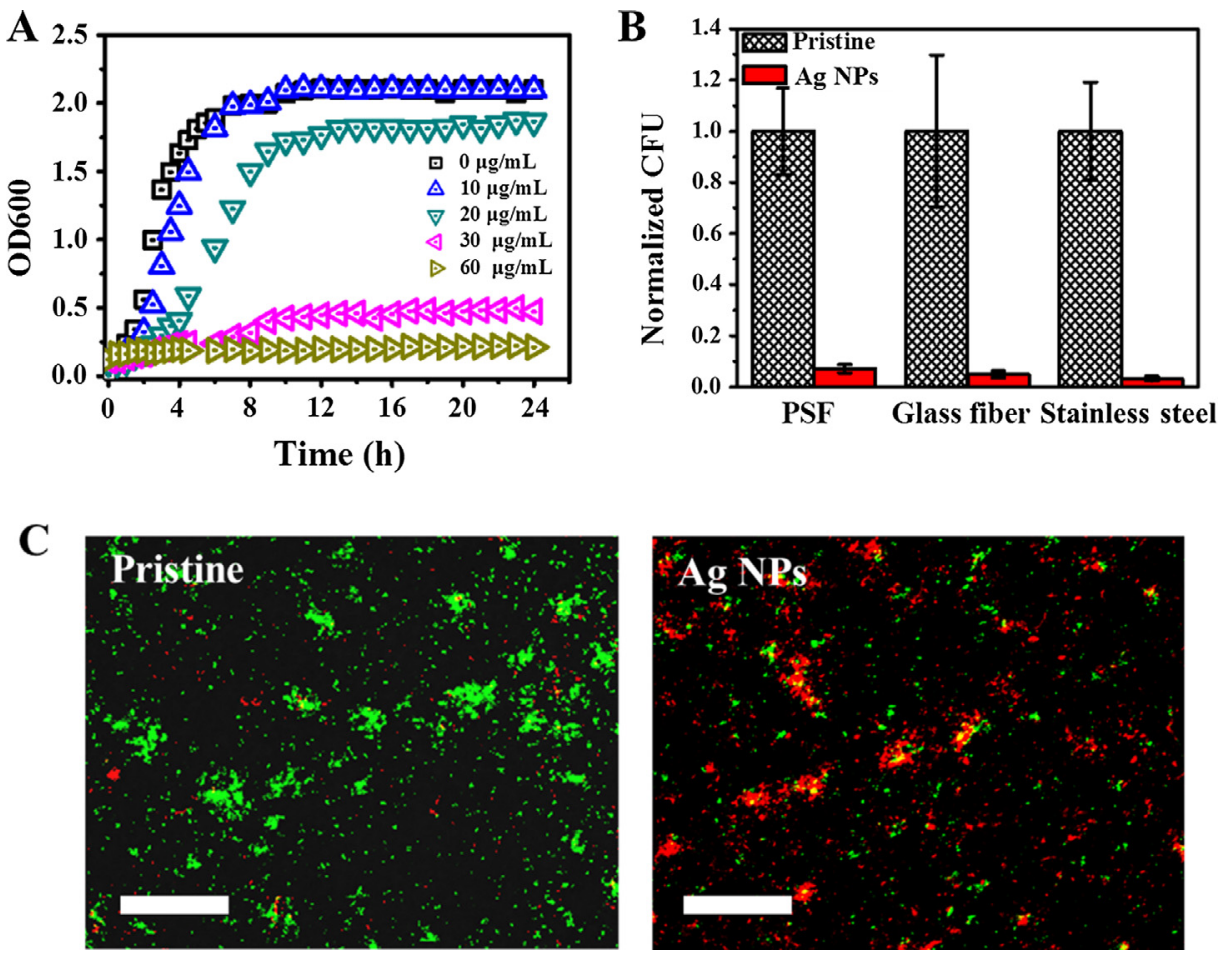

Fig. 5. Antibacterial properties of Ag NPs and Ag NPs containing membranes: (A) the growth curves of $E$. coli in LB media containing different concentrations of Ag NPs, (B) the normalized CFUs of live $E$. coli attached on the PSF UF membrane, glass fiber membrane and stainless steel before and after Ag NPs immobilization, and (C) representative CLSM images of live and dead $E$. coli cells attached on the pristine and silver containing PSF UF membrane stained with SYTO ${ }^{\circledR} 9$ and PI after $24 \mathrm{~h}$ incubation. The scale bars are $50 \mu \mathrm{m}$.

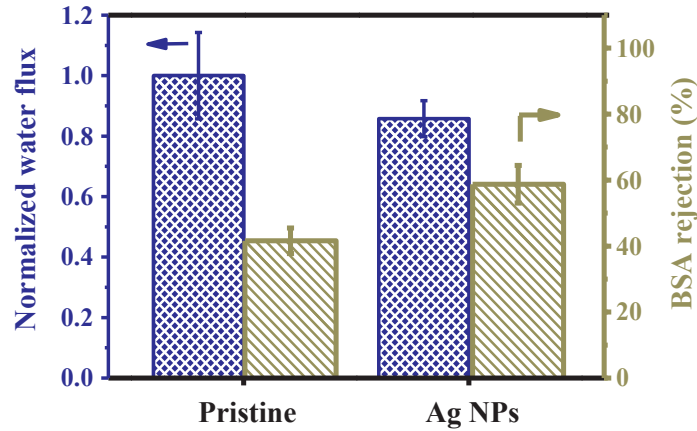

Fig. 6. Normalized water flux and BSA rejection of pristine and Ag NPs immobilized PSF UF membranes.

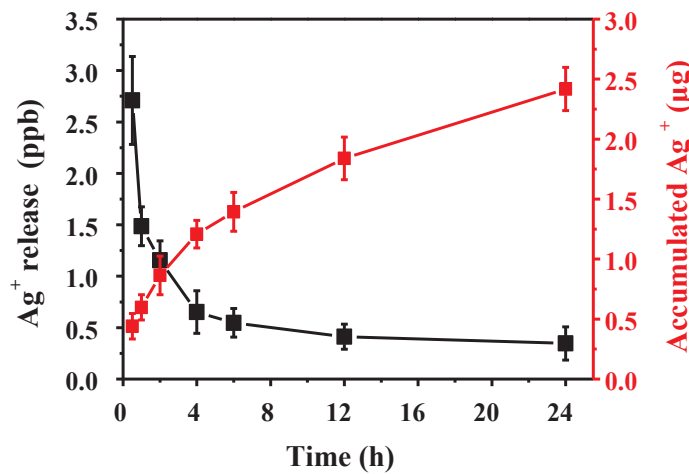

Fig. 7. The silver ions release profile from the PSF UF membranes and the accumulated silver ions in the water permeate over the filtration time.

to $0.35 \pm 0.16 \mathrm{ppb}$ in the $24 \mathrm{~h}$ of test with more water filtered through the membrane, as shown in Fig. 7. According to the U.S. Environmental Protection Agency [50], the maximal contaminant limit of silver ions in drinking water is $100 \mathrm{ppb}$, which is 35 times higher than the silver ion concentration in the water permeate, proving the safety of Ag NPs containing PSF UF membranes for water treatment. Moreover, during the filtration, the total accumulation of silver ions released from the membranes with $3.85 \mathrm{~cm}^{2}$ surface area was $2.4 \mu \mathrm{g}$, which is only $7 \%$ of its initial Ag NPs loading, indicating a very slow release of Ag NPs from the membrane surface. The morphologies of Ag NPs loaded PSF UF membranes before and after the filtration test were observed using SEM to quantify the number and size change of Ag NPs. As shown in Figs. S11 and S12, the average diameter of Ag NPs slightly decreases from $33.3 \pm 8.9 \mathrm{~nm}$ to $30.1 \pm 5.9 \mathrm{~nm}$ and the number of Ag NPs decreases from $153 \pm 9 \mu \mathrm{m}^{-2}$ to $132 \pm 7 \mu \mathrm{m}^{-2}$ after $24 \mathrm{~h}$ filtration test, which may illustrate that the silver leaching comes from both loosing particles and dissolution of the Ag NPs. Above all, all these results suggest that the silver leaching from the PSF UF membranes is very slow and there is no risk to environment with the application of membranes for water filtration.

\section{Conclusion}

In this study, a facile and universal approach has been developed to synthetize Ag NPs and simultaneously immobilize them onto diverse membranes for antibacterial properties. mPEG-SH was used as a ligand to slow down the reduction process of silver ions by dopamine to synthesize Ag NPs in a controllable manner and also to prevent the aggregation of Ag NPs in solution. Moreover, the Ag NPs could be in situ immobilized onto polysulfone ultrafiltration membrane, glass fiber membrane and stainless steel, regardless of their surface properties, but the size of Ag NPs was affected by the substrates. The formed Ag NPs were bigger in the bulk condition than on the membrane surfaces, and also the fibrous membrane surfaces provided a favorable surface to grow bigger Ag NPs than the flat membrane surface under the same conditions. In addition, our results find that the Ag NPs immobilization on the PSF UF membranes increased the membrane selectivity with higher BSA rejection but decreased membrane permeability with lower water flux in comparison with the pristine membrane. The Ag NPs containing membranes presented outstanding antibacterial properties with more than $90 \%$ antibacterial efficiency against both Gram-negative bacteria $E$. coli and Gram-positive bacteria $S$. aureus. The silver leaching study during the filtration test illustrates that the silver release from the PSF UF membranes was very slow and came from both loosing 
particles and dissolution of the Ag NPs. More importantly, results show that there is no risk to use Ag NPs incorporated membranes for water treatment. Future systematic investigation will focus on the dynamic filtration study to understand the antibiofouling performance and mitigation mechanism of Ag NPs incorporated membranes towards the filtration of bacterial-containing feed solution.

\section{Acknowledgement}

The authors gratefully acknowledge the funding support from National Natural Science Foundation of China (No. 21476249, No. 51708408), Chang-jiang Scholars and Innovative Research Team in the University of Ministry of Education, China (No. IRT-17R80), The Program for Innovative Research Team in University of Tianjin (No. TD13-5044) and The Science and Technology Plans of Tianjin (No. 17PTSYJC00060).

\section{Appendix A. Supplementary material}

The compositions of different reaction systems shown in Fig. 1 (Table S1); Surface charges of PSF UF membranes and stainless steel before and after Ag NP immobilization (Table S2); Photographs and UVVis spectra of the mixture solution at different reaction time (Figure S1); The TEM image of the synthesized Ag NPs (Figure S2); The hydrodynamic diameter distribution of Ag NPs in the solution before and after a week storage (Figure S3); Photographs of PSF UF membrane, glass fiber membrane and stainless steel before and after modification (Figure S4); The low magnification SEM image of Ag NPs loaded PSF UF membrane (Figure S5); The size distribution of Ag NPs generated on the PSF UF membrane, glass fiber membrane and stainless steel (Figure S6); The water contact angles of the PSF UF membranes with different immersion time (Figure S7); Diffusion inhibition zone of $E$. coli and $S$. aureus (Figure S8 and Figure S9); Normalized CFUs of live $S$. aureus attached on the PSF UF membrane, glass fiber and stainless steel before and after modification (Figure S10); The SEM image of Ag NPs loaded PSF UF membrane before and after the filtration test (Figure S11); The size and number of Ag NPs on the PSF UF membrane surfaces before and after the $24 \mathrm{~h}$ filtration test (Figure S12).

Supplementary data associated with this article can be found, in the online version, at https://doi.org/10.1016/j.apsusc.2018.06.066.

\section{References}

[1] A.G. Fane, R. Wang, M.X. Hu, Synthetic membranes for water purification: Status and future, Angew. Chem. Int. Ed. 54 (2015) 3368-3386.

[2] M.A. Shannon, P.W. Bohn, M. Elimelech, J.G. Georgiadis, B.J. Mariñas, A.M. Mayes, Science and technology for water purification in the coming decades, Nature 452 (2008) 301-310.

[3] E. Barzeev, F. Perreault, A.P. Straub, M. Elimelech, Impaired performance of pressure-retarded osmosis due to irreversible biofouling, Environ. Sci. Technol. 49 (2015) 13050-13058.

[4] H. Lin, P. Wei, M. Zhang, J. Chen, H. Hong, Z. Ye, A review on anaerobic membrane bioreactors: applications, membrane fouling and future perspectives, Desalination 314 (2013) 169-188.

[5] A. Bogler, S. Lin, E. Bar-Zeev, Biofouling of membrane distillation, forward osmosis and pressure retarded osmosis: principles, impacts and future directions, J. Membr. Sci. 542 (2017) 378-398.

[6] J. Mansouri, S. Harrisson, V. Chen, Strategies for controlling biofouling in membrane filtration systems: challenges and opportunities, J. Mater. Chem. 20 (2010) $4567-4586$.

[7] Y. Liu, Z. Liu, A. Zhang, Y. Chen, X. Wang, The role of EPS concentration on membrane fouling control: comparison analysis of hybrid membrane bioreactor and conventional membrane bioreactor, Desalination 305 (2012) 38-43.

[8] W. Yu, N. Graham, Y. Yang, Z. Zhou, L.C. Campos, Effect of sludge retention on UF membrane fouling: the significance of sludge crystallization and EPS increase, Water Res. 83 (2015) 319-328.

[9] R. Zhang, Y. Liu, M. He, Y. Su, X. Zhao, M. Elimelech, Z. Jiang, Antifouling membranes for sustainable water purification: strategies and mechanisms, Chem. Soc. Rev. 45 (2016) 5888-5924.

[10] J.-H. Li, B.-F. Yan, X.-S. Shao, S.-S. Wang, H.-Y. Tian, Q.-Q. Zhang, Influence of Ag/ TiO2 nanoparticle on the surface hydrophilicity and visible-light response activity of polyvinylidene fluoride membrane, Appl. Surf. Sci. 324 (2015) 82-89.
[11] L. He, L.F. Dumée, C. Feng, L. Velleman, R. Reis, F. She, W. Gao, L. Kong, Promoted water transport across graphene oxide-poly(amide) thin film composite membranes and their antibacterial activity, Desalination 365 (2015) 126-135.

[12] A. Tiraferri, C.D. Vecitis, M. Elimelech, Covalent binding of single-walled carbon nanotubes to polyamide membranes for antimicrobial surface properties, ACS Appl. Mat. Interfaces 3 (2011) 2869-2877.

[13] Q. Zhao, J. Hou, J. Shen, J. Liu, Y. Zhang, Long-lasting antibacterial behavior of a novel mixed matrix water purification membrane, J. Mater. Chem. A 3 (2015) $18696-18705$.

[14] L.F. Dumée, L. He, P.C. King, M.L. Moing, I. Güller, M. Duke, P.D. Hodgson, S. Gray, A.J. Poole, L. Kong, Towards integrated anti-microbial capabilities: novel biofouling resistant membranes by high velocity embedment of silver particles, $\mathrm{J}$. Membr. Sci. 475 (2015) 552-561.

[15] P.F. Andrade, A.F. de Faria, S.R. Oliveira, M.A. Arruda, M.C. Gonçalves, Improved antibacterial activity of nanofiltration polysulfone membranes modified with silver nanoparticles, Water Res. 81 (2015) 333-342.

[16] L. Tang, K.J.T. Livi, K.L. Chen, Polysulfone membranes modified with bioinspired polydopamine and silver nanoparticles formed in situ to mitigate biofouling, Environ. Sci. Technol. Lett. 2 (2015) 59-65.

[17] M. Ben-Sasson, X. Lu, E. Bar-Zeev, K.R. Zodrow, S. Nejati, G. Qi, E.P. Giannelis, M. Elimelech, In situ formation of silver nanoparticles on thin-film composite reverse osmosis membranes for biofouling mitigation, Water Res. 62 (2014) 260-270.

[18] B.L. Ouay, F. Stellacci, Antibacterial activity of silver nanoparticles: a surface science insight, Nano Today 10 (2015) 339-354.

[19] K.B. Holt, A.J. Bard, Interaction of silver(I) ions with the respiratory chain of Escherichia coli: an electrochemical and scanning electrochemical microscopy study of the antimicrobial mechanism of micromolar Ag +, Biochemistry 44 (2005) 13214-13223.

[20] W. Yang, C. Shen, Q. Ji, H. An, J. Wang, Q. Liu, Z. Zhang, Food storage material silver nanoparticles interfere with DNA replication fidelity and bind with DNA, Nanotechnology 20 (2009) 085102.

[21] X. Liu, L.-X. Foo, Y. Li, J.-Y. Lee, B. Cao, C.Y. Tang, Fabrication and characterization of nanocomposite pressure retarded osmosis (PRO) membranes with excellent antibiofouling property and enhanced water permeability, Desalination 389 (2016) $137-148$.

[22] F. Perreault, H. Jaramillo, M. Xie, M. Ude, M. Elimelech, Biofouling mitigation in forward osmosis using graphene oxide functionalized thin-film composite membranes, Environ. Sci. Technol. 50 (2016) 5840-5848.

[23] T. Yin, H.W. Walker, D. Chen, Q. Yang, Influence of $\mathrm{pH}$ and ionic strength on the deposition of silver nanoparticles on microfiltration membranes, J. Membr. Sci. 449 (2014) 9-14.

[24] J.H. Li, X.S. Shao, Q. Zhou, M.Z. Li, Q.Q. Zhang, The double effects of silver nanoparticles on the PVDF membrane: surface hydrophilicity and antifouling performance, Appl. Surf. Sci. 265 (2013) 663-670.

[25] S. Afkham, A. Aroujalian, A. Raisi, Fabrication of antimicrobial polyethersulfone microfiltration membranes by corona plasma-assisted coating silver nanoparticles, RSC Adv. 6 (2016) 108113-108124.

[26] A.Y. Booshehri, R. Wang, R. Xu, The effect of re-generable silver nanoparticles/ multi-walled carbon nanotubes coating on the antibacterial performance of hollow fiber membrane, Chem. Eng. J. 230 (2013) 251-259.

[27] Q. Liu, Z. Zhou, G. Qiu, J. Li, J. Xie, J.Y. Lee, A surface reaction route to increase the loading of antimicrobial Ag nanoparticles in forward osmosis membranes, ACS Sustain. Chem. Eng. 3 (2015) 2959-2966.

[28] L.F. Dumée, J.W. Maina, A. Merenda, R. Reis, L. He, L. Kong, Hybrid thin film nanocomposite membrane reactors for simultaneous separation and degradation of pesticides, J. Membr. Sci. 528 (2017) 217-224.

[29] J. Zhang, Y. Zhang, Y. Chen, L. Du, B. Zhang, H. Zhang, J. Liu, K. Wang, Preparation and characterization of novel polyethersulfone hybrid ultrafiltration membranes bending with modified halloysite nanotubes loaded with silver nanoparticles, Ind. Eng. Chem. Res. 51 (2012) 3081-3090.

[30] M. Sharma, P. Nagarajan, R. Sanjay, G. Madras, S. Bose, Facile one-pot scalable strategy to engineer biocidal silver nanocluster assembly on thiolated PVDF membranes for water purification, RSC Adv. 6 (2016) 38972-38983.

[31] J. Yin, Y. Yang, Z. Hu, B. Deng, Attachment of silver nanoparticles (AgNPs) onto thin-film composite (TFC) membranes through covalent bonding to reduce membrane biofouling, J. Membr. Sci. 441 (2013) 73-82.

[32] H. Lee, S.M. Dellatore, W.M. Miller, P.B. Messersmith, Mussel-inspired surface chemistry for multifunctional coatings, Science 318 (2007) 426-430.

[33] S.M. Kang, N.S. Hwang, J. Yeom, S.Y. Park, P.B. Messersmith, I.S. Choi, R. Langer, D.G. Anderson, H. Lee, One-step multipurpose surface functionalization by adhesive catecholamine, Adv. Funct. Mater. 22 (2012) 2949-2955.

[34] Z. Liu, Y. Hu, Sustainable antibiofouling properties of thin film composite forward osmosis membrane with rechargeable silver nanoparticles loading, ACS Appl. Mat. Interfaces 8 (2016) 21666-21673.

[35] Z. Yang, Y. Wu, J. Wang, B. Cao, C.Y. Tang, In situ reduction of silver by polydopamine: a novel antimicrobial modification of a thin-film composite polyamide membrane, Environ. Sci. Technol. 50 (2016) 9543-9550.

[36] N. Wang, T. Wang, Y. Hu, Tailoring membrane surface properties and ultrafiltration performances via self-assembly of polyethylene glycol-block-polysulfone-blockpolyethylene glycol block copolymer upon thermal and solvent annealing, ACS Appl. Mat. Interfaces 9 (2017) 31018-31030.

[37] Z. Liu, Y. Hu, C. Liu, Z. Zhou, Surface-independent one-pot chelation of copper ions onto filtration membranes to provide antibacterial properties, Chem. Commun. 52 (2016) 12245-12248.

[38] S. Agnihotri, S. Mukherji, S. Mukherji, Size-controlled silver nanoparticles synthesized over the range 5-100 $\mathrm{nm}$ using the same protocol and their antibacterial 
efficacy, RSC Adv. 4 (2014) 3974-3983.

[39] M.A. Pérez, R. Moiraghi, E.A. Coronado, V.A. Macagno, Hydroquinone synthesis of silver nanoparticles: a simple model reaction to understand the factors that determine their nucleation and growth, Cryst. Growth Des. 8 (2008) 1377-1383.

[40] R. Baron, M. Zayats, I. Willner, Dopamine-, L-DOPA-, adrenaline-, and noradrenaline-induced growth of Au nanoparticles: assays for the detection of neurotransmitters and of tyrosinase activity, Anal. Chem. 77 (2005) 1566-1571.

[41] F. Bernsmann, V. Ball, F. Addiego, A. Ponche, M. Michel, J.J.D.A. Gracio, V. Toniazzo, D. Ruch, Dopamine-melanin film deposition depends on the used oxidant and buffer solution, Langmuir 27 (2011) 2819-2825.

[42] C. Luo, Y. Zhang, X. Zeng, Y. Zeng, Y. Wang, The role of poly (ethylene glycol) in the formation of silver nanoparticles, J. Colloid Interface Sci. 288 (2005) 444-448.

[43] A. Shkilnyy, M. Soucé, P. Dubois, F. Warmont, M.-L. Saboungi, I. Chourpa, Poly (ethylene glycol)-stabilized silver nanoparticles for bioanalytical applications of SERS spectroscopy, Analyst 134 (2009) 1868-1872.

[44] I. Venditti, L. Fontana, I. Fratoddi, C. Battocchio, C. Cametti, S. Sennato, F. Mura, F. Sciubba, M. Delfini, M.V. Russo, Direct interaction of hydrophilic gold nanoparticles with dexamethasone drug: loading and release study, J. Colloid Interface
Sci. 418 (2014) 52-60.

[45] L. Fontana, M. Bassetti, C. Battocchio, I. Venditti, I. Fratoddi, Synthesis of gold and silver nanoparticles functionalized with organic dithiols, Colloids Surf., A 532 (2017) 282-289.

[46] A. Kumar, P.K. Vemula, P.M. Ajayan, G. John, Silver-nanoparticle-embedded antimicrobial paints based on vegetable oil, Nat. Mater. 7 (2008) 236-241.

[47] K. Kawai, T. Narushima, K. Kaneko, H. Kawakami, M. Matsumoto, A. Hyono, H. Nishihara, T. Yonezawa, Synthesis and antibacterial properties of water-dispersible silver nanoparticles stabilized by metal-carbon o-bonds, Appl. Surf. Sci. 262 (2012) 76-80.

[48] Z. Liu, L. Qi, X. An, C. Liu, Y. Hu, Surface engineering of thin film composite polyamide membranes with silver nanoparticles through layer-by-layer interfacial polymerization for antibacterial properties, ACS Appl. Mat. Interfaces 9 (2017) 40987-40997.

[49] M. Zhang, R.W. Field, K. Zhang, Biogenic silver nanocomposite polyethersulfone UF membranes with antifouling properties, J. Membr. Sci. 471 (2014) 274-284.

[50] H.T. Ratte, Bioaccumulation and toxicity of silver compounds: A review, Environ. Toxicol. Chem. 18 (1999) 89-108. 